\title{
Next generation network based carrier ethernet test bed for IPTV traffic
}

\author{
Fu, Rong; Berger, Michael Stübert; Zheng, Yu; Brewka, Lukasz Jerzy; Wessing, Henrik
}

Published in:

EUROCON 2009: INTERNATIONAL IEEE CONFERENCE DEVOTED TO THE 150 ANNIVERSARY OF ALEXANDER S. POPOV

Link to article, DOI:

10.1109/EURCON.2009.5167885

Publication date:

2009

Document Version

Publisher's PDF, also known as Version of record

Link back to DTU Orbit

Citation (APA):

Fu, R., Berger, M. S., Zheng, Y., Brewka, L. J., \& Wessing, H. (2009). Next generation network based carrier ethernet test bed for IPTV traffic. In EUROCON 2009: INTERNATIONAL IEEE CONFERENCE DEVOTED TO THE 150 ANNIVERSARY OF ALEXANDER S. POPOV (Vol. Vol 1-4, pp. 1781-1787). IEEE. https://doi.org/10.1109/EURCON.2009.5167885

\section{General rights}

Copyright and moral rights for the publications made accessible in the public portal are retained by the authors and/or other copyright owners and it is a condition of accessing publications that users recognise and abide by the legal requirements associated with these rights.

- Users may download and print one copy of any publication from the public portal for the purpose of private study or research.

- You may not further distribute the material or use it for any profit-making activity or commercial gain

- You may freely distribute the URL identifying the publication in the public portal 


\title{
NeXt Generation Network Based Carrier Ethernet Test Bed For IPTV TrafFiC
}

\author{
Rong Fu Member, Michael S Berger Member, Yu Zheng, Lukasz Brewka Member, Henrik Wessing \\ Member \\ Technical University of Denmark \\ Email: \{rofu, msbe, ljbr, hewe\}@fotonik.dtu.dk
}

\begin{abstract}
This paper presents a Carrier Ethernet (CE) test bed based on the Next Generation Network (NGN) framework. After the concept of CE carried out by Metro Ethernet Forum (MEF), the carrier-grade Ethernet are obtaining more and more interests and being investigated as the low cost and high performance services of transport network to carry the IPTV traffic. This test bed is approaching to support the research on providing a high performance carrier-grade Ethernet transport network for IPTV traffic.
\end{abstract}

Index Terms: Carrier Ethernet, Next Generation Network, Resource Admission Control, Network Protection

\section{INTRODUCTION}

With the emerging of highly demanding network services typically IPTV transport, network carriers are working hard to provide their transport network services with the carrier-class attributes such as assured end-to-end service of quality, large scalability and stable reliability, in the mean time, low cost and simple maintenance.

ITU-T IPTV and NGN focus groups at the same time have been working for years to develop the NGN architecture that provides a framework that allowing the delivering of network transport services. And it is now widely accepted that the Internet Protocol (IP) will form the basis for new services, as well as assist in the transition of circuit-based services to packet-based services (e.g. Voice and Video over IP). However, it is far from certain that IP routing technology will be adopted as the transport convergence layer. IP/MPLS has been widely deployed, especially in carrier backbone/core networks as a service layer and as a convergence layer, but Ethernet is fast becoming a credible alternative candidate. $95 \%$ of all data traffic either originates or terminates at Ethernet, and the data volume is forecast to grow tremendously given the impact of new video services and IPTV for example. This situation has prompted many telecom carriers to consider Ethernet as a potential convergence solution for NGN. With its scalability, ubiquity and natural support for IP services, Ethernet provides a compelling case. But before Ethernet can be adopted, it must be capable of supporting multiple services with at least the same level of quality as existing carrier services. In other words, Ethernet services must achieve a carrier-class of quality.

MEF brought out the Carrier Ethernet concept by means of adding the carrier-class services attributes on to the conventional Ethernet services. According to
MEF, a Carrier Ethernet service and the underlying equipment supporting the service must possess the following attributes: Standardized services, Scalability, Reliability, Quality of Service and Service management.

As a response to the increased interest in providing high performance network for delivering IPTV traffic, the HIPT project was founded with the objective of enhancing the carrier Ethernet transport for IPTV applications by developing technology that can fulfil the increasing requirements in terms of bandwidth and quality and at the same time reduce cost of network operation. As a phase toward the objective, a test bed based on the Carrier Ethernet is designed and built up for the purpose in providing a testing environment to optimize the IPTV transport network.

The following sections first introduce the Carrier Ethernet transport network architecture for IPTV in this HIPT project, followed by the design and functions description of this test bed. In the end, some results will be presented and discussion of future challenges in investigating into the Carrier Ethernet IPTV transport will be concluded.

\section{CARRIER ETHERNET ARCHITECTURE DESIGN}

The data services movement from the conventional voice services to the multimedia and video services gives great motivation to choose Carrier Ethernet as an important candidate of convergence services in the area of metro transport network. The SDH/SONET and ATM transport infrastructure which are dominating in this area before, however, cannot scale to support the rapid growth of the packet-switching data traffic in a cost effective manner. One of the SONET/SDH major limitations is the lack of flexibility and manageability in granularity of bandwidth so that smaller streams of traffic to the needs of individuals and enterprises cannot be managed through.

In another side, in the IP world, the IP/MPLS has been widely accepted in the area of core network. However, the IP based routing and signaling working mechanisms during the procedures such as routes discovery, resource reservation and network management gives a big limitation on the efficiency to precede the carrier-grade services.

Thus in HIPT, we investigate on carrying out a layer 2 Carrier Ethernet transport network architecture complied with Next Generation Network framework (Fig.1). 
Fig.1 is a NGN based transport network architecture which is provisioned by three function blocks: Service Control Functions (Service Control Function (Service Control Function in Fig.1), transport network control plane (RACF in Fig.1) and transfer functions (Layer 2 transport network in Fig.1). The architecture design scheme and mechanism are adopted with the ITU-T NGN architecture described in [1] and [2].

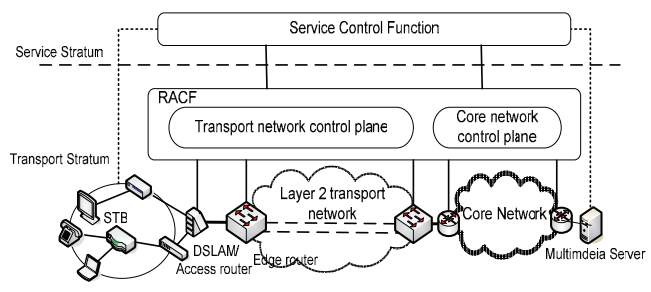

Fig.1 - Carrier Ethernet transport network architecture based on NGN architecture

Carrier Ethernet resides in the lowest block of Layer 2 transport network. Due to the cost-efficiency and scalability limitations described at the beginning of this section, the Layer 3 dynamic routing in the metro/access domain is replaced by a scalable architecture with static tunnels by means of PBB-TE or MPLS TP. According to the NGN architecture, there is centralized transport control plane above the transfer functions which deals with all the issues of transport network resource control and management. In Fig.1 this control plane refers to RACF (Resource Admission Control Functions) as the same name in [3]. The functions in the Service Control Function block are within network Service Stratum which is not connected directly to the transport network. It is located in the network service layer and deals with the application layer signaling, resource reservation negotiation, access authentication and accounting etc.

The following subsections are going to present and describe about this Carrier Ethernet transport network according to Fig.1.

\section{A. Carrier Ethernet transport network}

PBB-TE and MPLS TP are two popular Layer 2 transport technologies which can be selected as Carrier Ethernet candidates. The objective of MPLS TP is to abstract a subset of existing MPLS necessity in order to provide connection-oriented packet transport. Since IP/MPLS is considered as the main convergence technology within the core network, in our HIPT project, we chose MPLS TP as the Layer 2 transport technology by the reasons of its original attributes in compliance with IP/MPLS and also the benefits it brings we described as Carrier Ethernet in the previous sections.

As described in the previous section, the layer 2 transport technology MPLS TP avoids the IP routing procedure, but tries to set up Layer 2 data transfer MPLS tunnels to provide the end-to-end quality of service assured transfer services. According to MEF TS 10.1 [8], the MPLS TP based network distinguishes the services class by means of different LSPs. Additionally, in our project proposal, different traffic flows, which despite within the same service class will be assigned by different bandwidth profiles according to the network policy. In another words, there are different LSPs inside one MPLS tunnel which indicate one transport service and the transport direction, in the mean time, there will be more than one bandwidth profiles indicates the individual requirement for the network bandwidth carried by the LSPs. Thus, we takes the advantage of the flexibility of MPLS label stack - two labels will be assigned for each traffic flow, one for the destination and service class and the other one is for the bandwidth profile (Fig.2) This method deals well with the network scalability and bandwidth granularity problem in the large scale network such as MAN (Metro Area Network). This double label scheme is inspired from the PBB-TE. Therefore, in the Carrier Ethernet MPLS TP the network QoS assurance control mechanism is held within layer 2 label switching accompanied with statically label assignment. Compare to traditional IP/MPLS, it is much more efficient because the avoidance of the IP layer signaling and discovery procedures such as OSPF, RSVP.

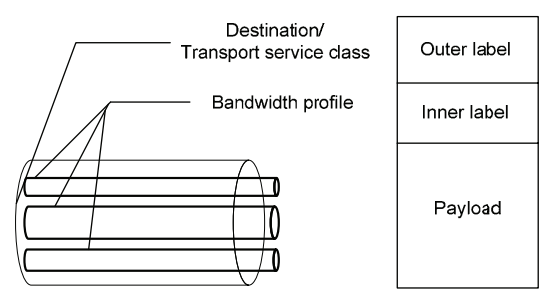

Fig.2 - Double label scheme in Carrier Ethernet

Thus, in the transport network in Fig.1 the end-to-end QoS is assured by assigning 2 layer labels to one traffic flow. The traffic flow will be assigned with the inner label when it arrives at the first label edge router (Edge router in Fig.1) to indicate the transport bandwidth profile, after that, in the Carrier Ethernet supported equipment (inside the Layer 2 transport network cloud in Fig.1), the traffic flow will be assigned with the outer label which explicit with the EVC and service class.

The functions such as MPLS label mapping and managing, network resource control and managing and policy decision are extracted and centralized inside the Carrier Ethernet control plane. In our design, it is referred to the term as RACF.

\section{B. Carrier Ethernet Control Plane}

MEF specifications for the Carrier Ethernet only 
concern the transport services and traffic behavior. To meet with the carrier-class transport services, a dynamic and intelligent control plane becomes a key area for future development. GMPLS control plane is considered most as the control plane for the Carrier Ethernet. It provides the dynamic IP based network QoS control and management. However, in HIPT project, we are introducing another candidate as transport network control plane RACF. Comparing to the more distributed GMPLS control plane, RACF is centralized on top of transport domain. It is designed as a single connect point between the service control functions and the transport network, in such a way to keep the underlying transport technology independent with the control technologies. In another aspect, the GMPLS control plane still adopts the set of IP routing, signaling and discovery protocols, the same layer 3 IP efficiency problems we mentioned at the beginning of this section can not really be solved by this way. Summarizing all the thoughts, RACF is investigated in our Carrier Ethernet control plane.

The RACF function block in Fig. 1 is compliance with the ITU standard [3]. According to the working scope, there are transport network control plane and core network control plane, while the functionalities are the same. Inside the RACF, there are two function entities: PD-FE (Policy Decision Function Entity) and TRC-FE (Transport Resource Control Function Entity). They provide the connection between the Service Control Functions in the service stratum and the Carrier Ethernet transport network. This single connection points maintains the network infrastructure simplicity and standardized which allow the underlying transport network working independently. The working procedure inside the RACF block refers to Fig.3

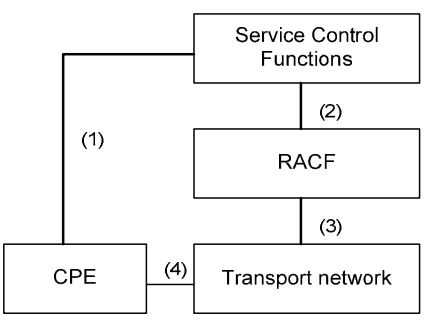

Fig. 3 QoS push model for Carrier Ethernet control plane

The control plane use a QoS push model to handle the QoS and network resource control for the transport network. (1) CPE initiates request (e.g. IPTV service) and sends it to service control functions. Service control functions processes the incoming requests and generates the network service request down to RACF through procedure (2). Upon receiving the service request from service control functions, the RACF will make the final policy decision and the network resource admission control by the means of consulting its own underlying network managing database and/ or inquiring the information to the according transport network. The final decision is made basing on the user profile, the network availability and also the policy rules installed by the network operators previously. After that, RACF will push the policy decision into the transport network (3) and the CPE then can starts to get its service according to the service class it ordered at the beginning.

Subsections A and B described the Carrier Ethernet transport technology and its control plane. The example recommendation from ITU-T can refer to [4]. Next section will present the design and architecture of such a Carrier Ethernet test according to the architecture introduced in this section.

\section{CARRIER ETHERNET TEST BED IMPLEMENTATION}

In order to approach more Carrier Ethernet research for HIPT project and reflect the status results on time. We built up the Carrier Ethernet test bed according to the Carrier Ethernet architecture we designed in the second section. Thus, a series of traffic performance test can be carried out within this test bed.

Fig. 4 is the architecture that has been implemented in this Carrier Ethernet test bed. It is compliance with the architecture in Fig. 1 and the functionalities are all compliance with the description in section II. The following subsections will present the test bed from the facilities, working procedures and demo results.

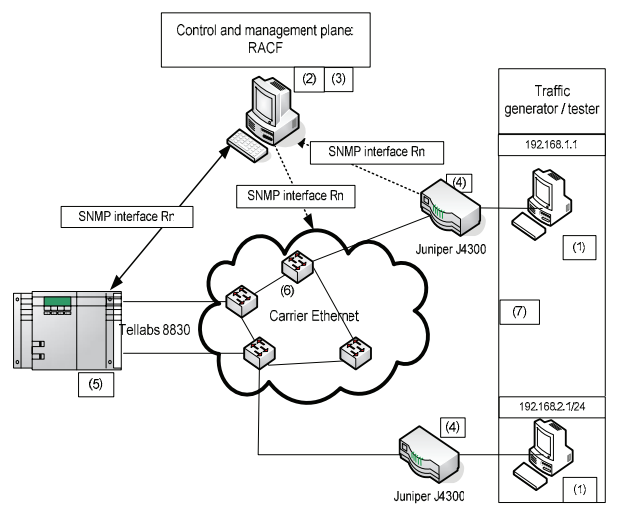

Fig.4 - Carrier Ethernet Test Bed architecture

\section{A. Test bed facilities}

Table 1 lists the equipments facilities for this Carrier Ethernet. The numbers following the function components name point out the component location in Fig.4. The description of each function component is given in the next subsection. 
Table 1 Test bed facility

\begin{tabular}{|l|l|}
\hline Function component & \multicolumn{1}{|c|}{ Equipment } \\
\hline Area border router (5) & $\begin{array}{l}\text { Tellabs 8830 Multi-service } \\
\text { router }\end{array}$ \\
\hline Access router (4) & Juniper 4300 \\
\hline CE switch (6) & $\begin{array}{l}\text { Tpack Carrier Ethernet } \\
\text { demo box }\end{array}$ \\
\hline Traffic generator & Aligent traffic generator \\
\hline
\end{tabular}

\section{B. Functional components}

In section II, it gives a brief description of the working scheme inside this Carrier Ethernet. In this section, we will give out the functions being implemented inside every function components inside this test bed.

(1) CPE: in this experimental network work as network service client. They locate as the end users behind the MPLS label edge router. It initiates network service requests with SIP signaling protocol. Based on SIP syntax, the service requests explicit different service classes and bandwidth requirements.

(2) Service Control Functions: As a SIP server, it analysis and routes the incoming SIP messages to the recipients. As part of the NGN transport network functions. SCF communicates with the network control plane to deal with the application level QoS negotiation.

(3) RACF: this function component emulates the functions as defined in NGN control plane RACF. The resource admission control functions are resides in this plane such as PD-FE, TRC-FE [3]. According to the network request, Control plane communicates with Carrier Ethernet equipments to push the policy and QoS parameters to config LSPs along the MPLS TP tunnels and get the network statistics through interface Rn.

(4) Edge router: 2 Juniper J4300 MPLS router are configured with LSPs of different service classes and represent as MPLS TP edge router.

(5) Area Border Router: As the border router, it can be seen as an extension point between both the control plane and other AS. It deals with the traffic policy control.

(6) Carrier Ethernet MPLS TP Switches: this CE supported switch is designed to provide switching within carrier-class. It supports up to 3 pushes and 3 pops per packet; MPLS TP OAM and LSP protection in hardware level. It receives the QoS and policy configuration command from RACF, thus each flow can be mapped to a separate output queue. It also supports the MEF bandwidth profile: $<$ CIR, CBS, EIR, $\mathrm{CF}, \mathrm{CM}>$ (refer to [8])

(7) Traffic generator and tester: It works as a service content provider or simulated VoIP client. It generates traffic with the parameters (traffic load, packet length, packets contents) configured according to the services it is required.

\section{Working procedures}

The working procedures within this Carrier Ethernet test bed loosely following the working procedures introduced by ITU-T's NGN recommendation in [1][2][3].

Before the session starts, each CE switch and edge router is configured manually with the MPLS LSP QoS and policy mapping information.

This test bed provides a JAVA simulated SIP client user interface (Fig. 5) from which user can customize the different scenarios by choosing the different destination IP addresses, service classes and bandwidths.

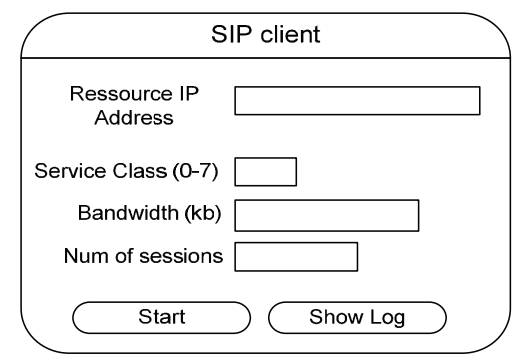

Fig. 5 SIP client user interface

In this test bed SIP client, More than one session can be set up simultaneously from one SIP client by specifying more than one session from the user interface.

After the session was triggered, a SIP based QoS service signaling goes between the two CPEs and service control functions. JAVA socket wrapped SIP based signaling carries on the session parameters of the ones input from the user interface. Upon receiving the request, Service control functions will invoke the RACF functions which are in charge of making the resource reservation decision according to the knowledge managed by RACF functions component. (Fig. 6)

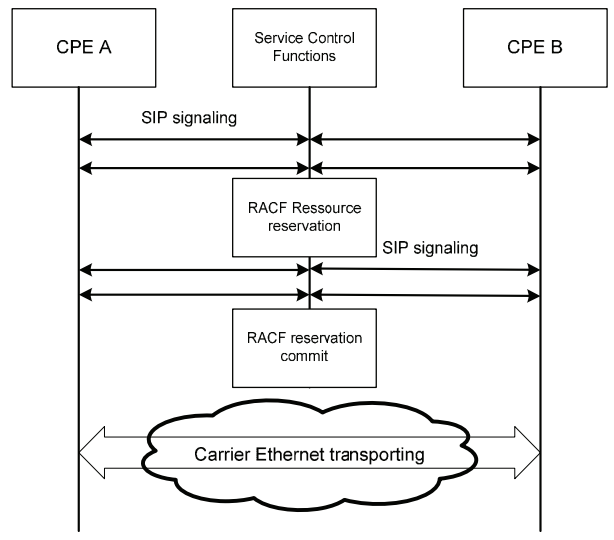

Fig. 6 Resource reservation and commit procedure 
The knowledge such as network topology and network resource is obtained by RACF function component by means of SNMP protocol through interface Rn. A JAVA API SNMP4J is used here as communication tool between the transport equipments and control function RACF server. By the end of a successful SIP signaling, RACF will commit its final resource reservation decision down to the edge Carrier Ethernet switches. This policy pushing is also by means of SNMP4J API. Thus, the QoS information interpreted through Service Control Functions and RACF function components are finally pushed into the CE devices: Carrier Ethernet switches.

In another word, until now, the SIP based session information is conversed into flow-based traffic parameters; each session is identified with a unique ID which is assigned at the beginning of the session initialization, and by this step, the session ID is mapped into a unique flow ID (Fig. 7)

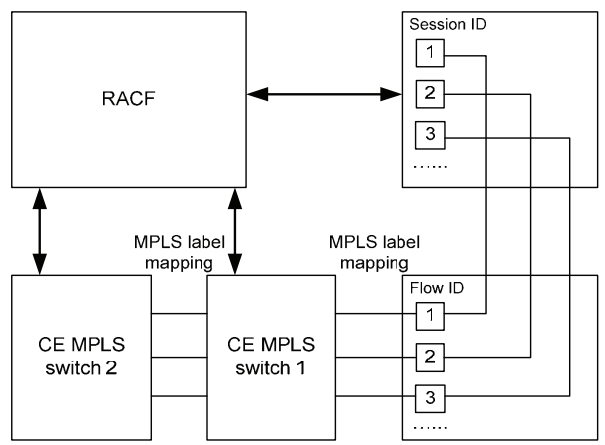

Fig. 7 Carrier Ethernet QoS and LSP mapping procedure

In our test bed the Carrier Ethernet MPLS switches support multiple ways of identifying the unique traffic flow. The unique flow ID can be derived from MPLS EXP bits, VLAN priority bits or IP DS bits. After receiving the policy parameters, all the $\mathrm{CE}$ switches will push the according MPLS label by checking the flow ID. Thus, the traffic flows can be treated classified.

\section{IV.RESULTS ANALYSIS}

Since this NGN based Carrier Ethernet architecture has been verified at a simulating research which the results have been published in another paper [14]. Thus, this architecture will be proved at emulating level within this test bed. We designed a test scenario based on the QoS traffic transport performance evaluation. The traffic generator will generator 3 traffic flows with the different IP address which indicating the different service class within the Carrier Ethernet network. All the three flows will be switch through the network as in Fig. 4 and be received by the traffic generator, where the classified service performance will be tested.

Thus, according to the working procedure in the last section, three LSP indicating the different service class and bandwidth profile will also be set up.

The bandwidth provision for the service classes and LSP bandwidth profile in Table 2 and Table 3 are compliance with the double label scheme we proposed in the Carrier Ethernet transport network section. The outer label indicates the service class as mentioned in Table 2, and the bandwidth profile for each flow is decided in Table 3

Table 2 service class provision

\begin{tabular}{|c|l|}
\hline Service class & Bandwidth (Kpbs) \\
\hline 0 & 4900 \\
\hline 1 & 4900 \\
\hline 2 & 4900 \\
\hline 3 & 4900 \\
\hline
\end{tabular}

Table 3 LSP bandwidth profile

\begin{tabular}{|c|l|}
\hline Flow & Bandwidth profile \\
\hline RT (class 0) & $\begin{array}{l}\text { Peak data rate: } 4000 \mathrm{k} \\
\text { Commited data rate: } 4000 \mathrm{k}\end{array}$ \\
\hline Video (class 2) & Peak data rate: $4000 \mathrm{k}$ \\
\hline BestEffort (class3) & No commited data rate \\
\hline
\end{tabular}

Fig. 8 contains three plots: Seq Errors counts the sequence number error for every incoming packet in each traffic flow; Tx Test Throughput shows the traffic throughput coming out from the traffic generator and the Rx Test Throughput shows the flows throughput incoming.
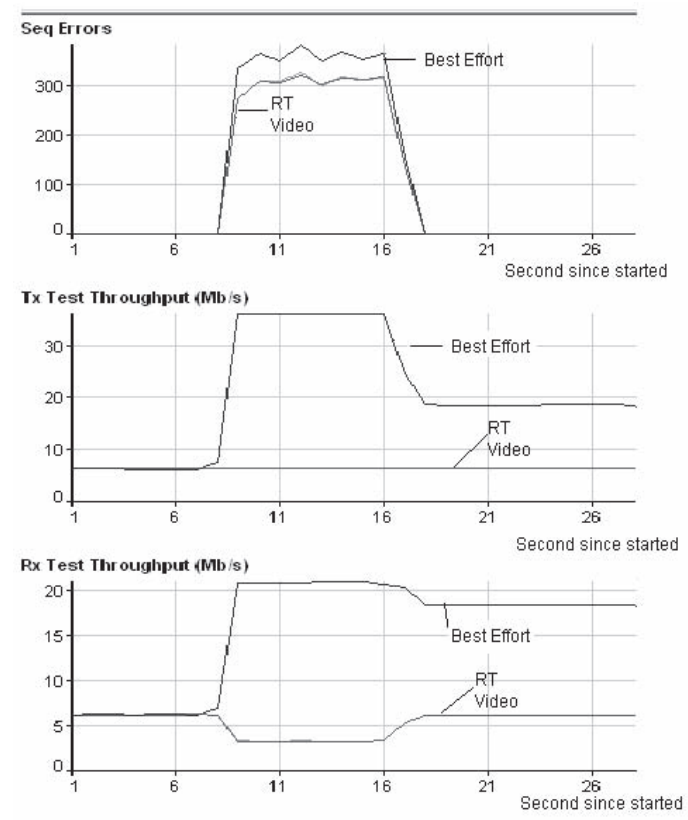

Fig. 8 traffic classified performance and throughput

From these three plots we can well tell, that the flow with highest service class (RT) can always get the assurance under their committed data rate which the 
lowest one (BestEffort) is always be sacrificed. In the "hill" part of the second and the third plots, the Tx and $\mathrm{Rx}$ throughput has the same trend but different scale. The BestEffort traffic has to be dropped to make the other two flows keep their committed bandwidth profile. However from the Seq Errors plot we can also see the packets dropping from the two higher classes, that is because the total bandwidth far exceeds the total bandwidth provision for the certain service classes. The in total bandwidth consumption is beyond the capacity of the network, so the even the first class traffic need to be dropped.

This classified performance is a demonstration about the Carrier Ethernet QoS control. More attributes are already undergoing as our future challenges and demonstration goals in HIPT project.

\section{FUTURE CHALLENGES}

This Carrier Ethernet test bed has just been through its first stage, only basic Carrier Ethernet transport functions are set up and emulated. However, it is not enough to be able to carry the IPTV traffic with the end-to-end QoS under the basic transport functions and control mechanisms. Here we propose two study points we are working on.

\section{A.OAM for IPTV flow monitoring}

With the IPTV services, the viewers will directly experience the quality of the network, thus it is very important that the network delivers carrier-class quality of service. The networks do not deliver the required OAM functionalities and IPTV operators are forced to deploy very expensive solutions to monitor the TV signals. MPLS network OAM is specific to the transport network and functionality is referenced from ITU-T's Y.1711 [9]. This provides the same OAM concepts and methods (e.g. connectivity verification, alarm suppression, remote defect indication) already available in other transport networks, without requiring complex IP data plane capabilities. Ongoing standardization initiatives focus on G.8113 [10] and G.8114 [11].

In this HIPT project, both point-to-point and point-to-multipoint OAM will be considered to run within our Carrier Ethernet network to support the carrier-class network survivability.

\section{B. Resiliency and survivability}

MPLS TP therefore defines its protection capability using ITU-T G.8121/Y.1382 [12] and G.8132/Y.1382 [13]. MPLS fast ReRoute Network survivability traditionally deals with connection recovery after infrastructure or equipment failures, e.g. cable cuts or node outages, which are characterized by loss of signal. In addition to these "hard failures", users of IPTV may experience signal quality degradation as a "soft failure", caused by gradual component degrading or malfunction. Since the customers' perception of the signal quality is critical for the successfulness of IPTV, survivability measures related to "soft failure" will be investigated in HIPT project. Accordingly in this test bed, the Carrier Ethernet access switches inside the Carrier Ethernet clouds supports the carrier-class OAM and protection. A set of failure scenarios could be set up to measure the reaction time within the carrier-class in order to improve the experience of services. Furthermore, multicast IPTV services with survivability will also be test within this test bed.

\section{ACKNOWLEDGEMENT}

The HIPT project is funded by Danish National Advanced Technology Foundation.

\section{CONCLUSION}

The work has been done with the NGN based Carrier Ethernet test bed provides a good demonstrating model and testing environment for the research on Carrier Ethernet. First, it demonstrates the idea that deploys Carrier Ethernet as transport service layer in the NGN transport network. Furthermore it verifies the advantages of this Carrier Ethernet architecture. It also carried out a contribution to design the RACF as transport control plane for this Carrier Ethernet. Most important, this test bed provides a testing environment for the future studies on achieving the carrier-grade attributes of more transport services.

\section{REFERENCES}

[1]. ITU-T Rec. Y.2001 "Next Generation Network Frameworks and functional architecture models, General overview of NGN" December 2004

[2]. ITU-T Rec. Y.2011 "General principles and general reference model for Next Generation Network" October 2004

[3]. ITU-T Rec. Y.2111 "Resource and admission control functions in Next Generation Networks" September 2004

[4]. ITU-T Y.2112, “A QoS Control Architecture for Ethernet-based IP Access Networks"

[5]. IEEE Std. 802.1Q-2005: "Virtual Bridged Local Area Networks".

[6]. IEEE Std. 802.1ad-2005: "Virtual Bridged Local Area Networks"

[7]. Metro Ethernet Forum Technical Specification MEF 6.1: "Ethernet Services Definitions -- Phase 2"

[8]. Metro Ethernet Forum Technical Specification MEF10.1: "Ethernet Services attributes Phase 2"

[9]. ITU-T Y.1711 "OAM mechanism for MPLS networks"

[10].ITU-T G.8113 "Requirements for OAM function in T-MPLS based networks"

[11].ITU-T G.8114 "Operation and maintenance mechanism for T-MPLS layer networks"

[12].ITU-T G.8121/Y.1382 “ T-MPLS linear protection switching with $1+1,1: 1$ and $1: N$ options" 
[13].ITU-T G.8132/Y.1382 “T-MPLS ring protection switching"

[14]. Rong Fu, Yanmeng Wang, Michael S.Berger: "Carrier Ethernet control plane based on the Next Generation Network ” ITU-T Kaleidoscope conference, 2008

Rong Fu: was born in 1982, she got her M.Sc. EE from the Technical University of Denmark in 2007 and is working as a $\mathrm{PhD}$ student in the same school until now. She is working involve with the HIPT project and main research on the control plane for Carrier Ethernet and the IPTV traffic end-to-end QoS delivery through multiple domains.

Michael S.Berger: was born in 1972 and received the M.Sc. $\mathrm{EE}$ and $\mathrm{PhD}$ from the Technical University of Denmark in 1998 and 2004. He is currently Associate Professor at the university within the area of switching and network node design. In his $\mathrm{PhD}$ thesis "Architectures of Electro-Optical Packet Switched Networks", focus was on network- and node architecture for future packet switched networks. Currently, he is leading the HIPT project on next generation IP networks partly funded by the Danish National Advanced Technology Foundation.

Lukasz Brewka: was born in 1981 and received the M.Sc. EE from the Technical University of Denmark in 2008, now he is pursuing his $\mathrm{PhD}$ at the same university. He is involved in the European ICT project Alpha and his main research activities concern Inter-domain QoS provisioning.

Henrik Wessing: After completing the Master degree, Henrik Wessing worked as research assistant in the Networking Competence Area at Research Center COM (now DTU Fotonik), and in 2001 he began to pursuit his $\mathrm{PhD}$ studies on electronic control of optical infrastructures and components. After completing his Ph.D., Henrik Wessing continued at DTU Fotonik, with responsibility for the coordination, maintenance and development of research activities related to the experimental platform. Currently he is also involved in the European ICT project ALPHA coordinating the DTU activities and in the HIPT project as WP leader for demonstration activities. 